\title{
Review: dietary advice leads to a modest reduction in blood cholesterol concentration
}

\author{
Tang JL, Armitage JM, Lancaster T, et al. Systematic review of dietary intervention trials to lower blood total cholesterol in \\ free-living subjects. BMJ 1998 Apr 18;316:1213-20.
}

\section{Questions}

Can dietary advice help people to lower their blood total cholesterol concentration? Which type of diet is most effective?

\section{Data sources}

Studies were identified by searching Medline, Human Nutrition, EMBASE/Excerpta Medica, and Allied and Alternative Medicine; handsearching the American Journal of Clinical Nutrition; scanning the references of relevant trials; and contacting experts.

\section{Study selection}

Studies published before 1996 were selected if they had $\geqslant 2$ groups and included a control group, used random allocation, tested a global dietary modification, and measured lipid concentrations. Exclusion criteria were specific supplementation diets, multifactorial intervention trials, trials of lowering body weight or blood pressure, interventions of $<4$ weeks, and randomisation of workplaces or general practices.

\section{Data extraction}

2 reviewers extracted data on type of diet, compliance, mean change in blood total cholesterol concentration, and amount of advice given. The 4 categories of diets were step 1 and 2 diets of the American Heart Association (AHA) which lowered the total intake of fat and increased the ratio of polyunsaturated to saturated fat (the step 2 diet was more intensive than the step 1 diet); diets that increased the ratio of polyunsaturated to saturated fat with little or no change in total fat content; and low total fat diets that did not change the proportions of types of fat consumed.

\section{Main results}

19 of 133 randomised controlled trials met the selection criteria and yielded 28 comparisons. Follow up ranged from 6 weeks to 5 years. 8 studies ( 8 comparisons) and 5 studies (9 comparisons) used the AHA step 1 and 2 diets, respectively; 6 studies (7 comparisons) used diets that increased the polyunsaturated to saturated fat ratio; and 4 studies (4 comparisons) used diets that reduced total fat intake. 2 studies used $>1$ type of diet. Dietary advice led to a reduction in the overall weighted mean blood total cholesterol concentration and this effect was maintained across the 4 categories of diet; the AHA step 2 dietary advice and dietary advice that aimed to increase the ratio of polyunsaturated to saturated fat were more effective than the AHA step 1 dietary advice (table). Heterogeneity existed among all trials $(\mathrm{p}<0.0001)$, trials of AHA step 2 diets $(\mathrm{p}<0.001)$, and trials of diets that changed fat ratios $(\mathrm{p}<0.001)$.

\section{Conclusions}

Dietary advice leads to a modest reduction in blood total cholesterol concentration. More intensive dietary advice that includes advice about increasing the polyunsaturated to saturated fat ratio is most effective.

Dietary advice $v$ control for mean percentage change in the blood total cholesterol concentration at up to 5 years follow up

\begin{tabular}{ll}
\hline Study group & Weighted mean reduction $(95 \%$ CI) \\
\hline All studies* & $5.7 \%(5.2$ to 6.3$)$ \\
AHA step 1 diets & $3.0 \%(1.8$ to 4.1$)$ \\
AHA step 2 diets* & $5.6 \%(4.7$ to 6.5$)$ \\
Change in fat ratio diets* & $7.6 \%(6.2$ to 9.0$)$ \\
Reduction in total fat diets & $5.8 \%(3.8$ to 7.8$)$ \\
\hline
\end{tabular}

$\mathrm{AHA}=$ American Heart Association.

*Heterogeneity existed among trials.

Sources of funding:Department of Health and Medical Research Council;British Heart Foundation; Imperial Cancer Research Fund.

For correspondence: Dr J M Armitage, Senior Research Fellow, Clinical Trial Service Unit, Radcliffe Infirmary, Oxford OX2 6HE,UK. Fax +44 (0)1865 404821.

\section{Commentary}

The use of lipid lowering agents for people at risk of coronary heart disease (CHD) mortality has already been proved. ${ }^{1}$ However, these agents are costly and there has been much discussion about the effectiveness of dietary modification to reduce cholesterol concentrations. A previous review showed that dietary changes reduced cholesterol concentrations by $10-15 \%$, but these studies took place in controlled situations where dietary compliance could be almost guaranteed..$^{2}$ These results cannot be translated to real life situations where compliance is an issue. The study by Tang et $a l$ is a well conducted review. They included only trials of people who lived in the community, and the results can therefore be translated into practice. The authors were not able to find any unpublished studies. Of note is the substantial degree of heterogeneity among the effects produced in different studies, which may be caused by differences in the intensity and type of intervention, characteristics of the study population, or completeness and duration of follow up.

This review is of interest to nurses who care for patients with an increased risk of CHD mortality. Its results suggest that dietary advice will reduce blood total cholesterol by $3-8 \%$, depending on the type and intensity of the diet advocated. Dietary compliance was clearly an issue as the dietary targets were only met in a few of the studies. More research is required to develop better methods of communicating dietary advice and maintaining compliance with the advice. It is also worth noting that Tang et al excluded studies that evaluated dietary advice given together with other interventions. People who are at greater risk of CHD are likely to have several risk factors that may need modifying. Advice on lowering cholesterol concentrations is therefore unlikely to be given in isolation.

Kate Johnson, RGN, MA
Cardiac Liaison Nurse
London, UK
Royal Brompton and Harefield NHS Trust
Randomised trial of cholesterol lowering in
4444 patients with coronary heart disease: the
Scandinavian Simvastatin Survival Study (4S).
Lancet 1994;344:1383-9.
Clarke R, Frost C, Collins R, et al. BMJ
1997;314:112-17.

Kate Johnson, RGN, MA d NHS Trust 1997:314:112-17. Collins R, et al. BMJ 